\title{
Applying Machine Learning Algorithms to Segment High-Cost Patient Populations
}

\author{
Jiali Yan, $M S^{7}$, Kristin A. Linn, $P h D^{2}$, Brian W. Powers, MD, MBA $A^{3,4,5,6}$, Jingsan Zhu, MS, MBA ${ }^{7}$, \\ Sachin H. Jain, MD, MBA ${ }^{5}$, Jennifer L. Kowalski, $M S^{8}$, and Amol S. Navathe, MD, PhD ${ }^{7,9}$
}

'Department of Medicine, University of Pennsylvania Perelman School of Medicine, Philadelphia, PA, USA; ${ }^{2}$ Department of Biostatistics, Epidemiology, and Informatics, University of Pennsylvania Perelman School of Medicine, Philadelphia, PA, USA; ${ }^{3}$ Department of Medicine, Brigham and Women's Hospital, Boston, MA, USA; ${ }^{4}$ Department of Population Medicine, Harvard Medical School/Harvard Pilgrim Health Care Institute, Boston, MA, USA; ${ }^{5}$ CareMore Health System, Cerritos, CA, USA; ${ }^{6}$ Atrius Health, Boston, MA, USA; 7 Department of Medical Ethics and Health Policy, University of Pennsylvania Perelman School of Medicine, Philadelphia, PA, USA; ${ }^{8}$ Anthem Public Policy Institute, Washington, DC, USA; ${ }^{9}$ Corporal Michael J. Cresencz VA Medical Center, Philadelphia, PA, USA.

BACKGROUND: Efforts to improve the value of care for high-cost patients may benefit from care management strategies targeted at clinically distinct subgroups of patients.

OBJECTIVE: To evaluate the performance of three different machine learning algorithms for identifying subgroups of high-cost patients.

DESIGN: We applied three different clustering algorithms - connectivity-based clustering using agglomerative hierarchical clustering, centroid-based clustering with the $\mathrm{k}$-medoids algorithm, and density-based clustering with the OPTICS algorithm-to a clinical and administrative dataset. We then examined the extent to which each algorithm identified subgroups of patients that were (1) clinically distinct and (2) associated with meaningful differences in relevant utilization metrics.

PARTICIPANTS: Patients enrolled in a national Medicare Advantage plan, categorized in the top decile of spending $(n=6154)$.

MAIN MEASURES: Post hoc discriminative models comparing the importance of variables for distinguishing observations in one cluster from the rest. Variance in utilization and spending measures.

KEY RESULTS: Connectivity-based, centroid-based, and density-based clustering identified eight, five, and ten subgroups of high-cost patients, respectively. Post hoc discriminative models indicated that density-based clustering subgroups were the most clinically distinct. The variance of utilization and spending measures was the greatest among the subgroups identified through density-based clustering.

CONCLUSIONS: Machine learning algorithms can be used to segment a high-cost patient population into subgroups of patients that are clinically distinct and

Jiali Yan and Kristin A. Linn contributed equally to this work.

Prior Presentation(s) This study was presented, in part, at Academy Health; June 25, 2018; Seattle, Washington.

Electronic supplementary material The online version of this article (https://doi.org/10.1007/s11606-018-4760-8) contains supplementary material, which is available to authorized users.

Received September 14, 2018

Revised October 30, 2018

Accepted November 16, 2018

Published online December 12, 2018 associated with meaningful differences in utilization and spending measures. For these purposes, density-based clustering with the OPTICS algorithm outperformed connectivity-based and centroid-based clustering algorithms.

KEY WORDS: high-cost patients; machine learning; patient segmentation.

J Gen Intern Med 34(2):211-7

DOI: $10.1007 / \mathrm{s} 11606-018-4760-8$

(c) Society of General Internal Medicine 2018

\section{INTRODUCTION}

Efforts to improve the value of care for high-cost patients may benefit from care management strategies targeted at clinically distinct subgroups of patients ${ }^{1-4}$. Existing frameworks for segmenting high-cost patients are derived from expert opinion and are based predominately on patterns of comorbidity and functional status $1,3,5,6$. There may be an opportunity to supplement these approaches using machine learning methods.

Clustering is an unsupervised machine learning technique that groups observations (e.g., patients) according to similarities among measured characteristics. There are a variety of approaches to clustering. Three of the most commonly used are connectivity-based clustering (also known as hierarchical clustering), centroid-based clustering, and density-based clustering ${ }^{7,8}$. Connectivity-based clustering algorithms sequentially combine, or split, groups of observations based on a metric reflecting the similarity among observations. Centroidbased clustering algorithms begin with a pre-specified number of subgroups and then assign observations to the subgroup with the closest centroid based on a distance metric. Common centroid-based clustering algorithms include k-means and kmedoids. Density-based clustering algorithms identify areas of varied density within a dataset and group together observations that make up areas of high density. One popular densitybased clustering algorithm is ordering points to identify the clustering structure (OPTICS), which is an extension of the earlier density-based spatial clustering of applications with noise (DBSCAN) algorithm. Table 1 provides a broad 
overview of these three clustering approaches and summarizes selected advantages and disadvantages of each.

Clustering has been used to identify novel disease subgroups across various conditions, including asthma 9,10 , $\mathrm{COPD}^{11,12}, \mathrm{CHF}^{13,14}$, and neurologic disorders ${ }^{15,16}$. However, the application of clustering to health care delivery remains nascent ${ }^{17,18}$. In this study, we aimed to evaluate the performance of connectivity-based, centroid-based, and density-based clustering for identifying subgroups of highcost patients using a clinical and administrative dataset.

\section{METHODS}

\section{Study Population and Data}

The study population consisted of patients enrolled in a national Medicare Advantage plan who were in the top decile of total per-patient spending in $2014(n=6154)$. See an accompanying article for a full description of the study population ${ }^{19}$. We extracted demographic and clinical data directly from the health plan's electronic data warehouse (EDW). Data were obtained for the years 2013-2015. We grouped 377 unique variables into several major categories: demographics, chronic conditions, active diagnoses, procedures, laboratory, and pharmacy. A full list and description of the study variables is provided in Appendix 1.

\section{Data Pre-processing}

An important first step in analyzing datasets with large numbers of variables is to remove redundancies and correlations that limit the ability to extract meaningful information. Since we aimed to identify clinically distinct subgroups of patients, we sought to remove variables that would not provide meaningful differentiation among patients. First, we removed variables with extremely low variance based on two criteria: (1) the ratio of the second most common value to the most common value was $\geq 0.99$ and (2) the percentage of unique values (the number of unique values divided by the number of observations) was $\leq 1 \%$. A total of 214 (of 377) variables were removed based on these criteria. Next, we removed non-binary variables that were highly correlated with others. We defined high correlation as a Pearson correlation coefficient greater than 0.85 . A total of 2 (of 163) variables were removed based on this criterion. A complete list of the remaining 161 variables and a description of a standard method used for imputing missing values are provided in Appendices 2 and 3, respectively.

\section{Dimension Reduction}

When clustering datasets with large numbers of variables (i.e., dimensions), a common task is to reduce the number of variables in the dataset while retaining as much of the original information and structure as possible, a task known as dimension reduction. Dimension reduction not only reduces computational burden but also mitigates the "curse of dimensionality"- the fact that data become increasingly sparse as the number of variables increases ${ }^{20,21}$. The curse of dimensionality makes it conceptually and computationally difficult to converge on a reasonable clustering solution.

We utilized a validated, non-linear dimension reduction algorithm called t-distributed stochastic neighbor embedding (t-SNE) ${ }^{22}$. The t-SNE method takes as input a highdimensional data set and maps each observation to a lowerdimensional space. We ran a specific implementation of t-SNE known as the Barnes-Hut algorithm and mapped to a twodimensional space in order to facilitate visualization ${ }^{22,23}$. Full details on t-SNE implementation are provided in Appendix 4.

\section{Model Tuning}

For each of the algorithms described below, we followed a standardized approach for tuning model parameters. First, we a priori restricted algorithm solutions to those that yielded between five and ten clusters. This helped to ensure that the resultant subgroups of patients would not be so small as to be operationally insignificant. Next, we calculated the average silhouette width for each solution. Average silhouette width, which we calculated using Euclidean distance, reflects how similar an observation is to those in its own cluster as compared to observations in other clusters ${ }^{24}$. Finally, we selected the solution that maximized average silhouette width. Additional information on model tuning is provided in Appendix 5 .

\section{Connectivity-Based Clustering}

For connectivity-based clustering, we used agglomerative hierarchical clustering with Ward's criterion ${ }^{25}$. This algorithm places each observation in its own cluster and then sequentially merges the two most similar clusters until there is only one large group. The decision of which clusters to merge at each step is made by selecting the combination that minimizes the squared Euclidean distance (e.g., the "ordinary" straight-line distance) between observations within each cluster. The result is a hierarchy, or dendrogram, that classifies each observation as a member of progressively larger clusters. Cutting the hierarchy at different levels, therefore, generates a different number of final clusters. We cut the hierarchy at six different levels to produce solutions with five to ten clusters. We then computed the average silhouette width for all observations and selected the clustering with the highest value.

\section{Centroid-Based Clustering}

For centroid-based clustering, we used the k-medoids algorithm ${ }^{26}$. K-medoids requires pre-specification of the number of clusters $(k)$ within which to group observations. The algorithm begins with a random selection of $k$ observations to serve as the center, or medoid, of each cluster. Observations are then assigned to the cluster with the closest medoid, as defined by a chosen distance metric (e.g., Euclidean). Next, the algorithm re-selects the most central observation for each cluster and conducts a re-assignment of observations until it 
Table 1 Overview of Common Clustering Methods Used in This Study

\begin{tabular}{|c|c|c|c|c|}
\hline Method & Algorithm & Computational approach & Advantages & Disadvantages \\
\hline $\begin{array}{l}\text { Connectivity- } \\
\text { based }\end{array}$ & $\begin{array}{l}\text { Agglomerative } \\
\text { hierarchical clustering } \\
\text { with Ward's criterion }\end{array}$ & $\begin{array}{l}\text { Clusters are sequentially merged } \\
\text { according to a chosen distance } \\
\text { metric to form a hierarchy. }\end{array}$ & $\begin{array}{l}\text { - Does not require a priori } \\
\text { specification of the } \\
\text { number of clusters. } \\
\text { - Works with a variety of } \\
\text { distance metrics and } \\
\text { linkage methods to merge } \\
\text { clusters. } \\
\text { - Standard methods exist } \\
\text { to be able to visualize the } \\
\text { entire cluster hierarchy. }\end{array}$ & $\begin{array}{l}\text { - High computational cost with } \\
\text { high-dimensional data such that } \\
\text { obtaining a clustering solution may } \\
\text { be infeasible. } \\
\text { - Requires specifying what level to } \\
\text { split the hierarchy to extract a final } \\
\text { clustering. } \\
\text { - Does not allow for outlier } \\
\text { observations (i.e., forces every } \\
\text { observation into a cluster) }\end{array}$ \\
\hline
\end{tabular}

Centroid- K-medoids

based

$\begin{array}{ll}\text { Density-based } & \begin{array}{l}\text { Ordering points to } \\ \text { identify the clustering } \\ \text { structure (OPTICS) }\end{array}\end{array}$

Assigns subjects to the nearest medoid, defined as the most central observation within a cluster.
- Simple, fast.

- Works with a variety of distance metrics.

- More robust to outliers than other centroid-based approaches, such as k-means.

- More memory intensive than other
centroid-based approaches, such as
k-means.
- Must specify the number of
clusters a priori or evaluate a large
number of different clustering
solutions to choose the number of
clusters.
- Does not allow for outlier
observations (i.e., forces every
observation into a cluster)
- Requires specification of multiple
tuning parameters that are not
intuitive.
- Produces noise points (can also be
considered an advantage).
- May fail to converge in some
cases if there is large variance in the
density of clusters.

Orders observations based on information about their nearest neighbors and defines clusters as areas with a high density of data points.

\author{
- Robust to outliers, with \\ the ability to label them as \\ noise points. \\ - Can return clusters of \\ arbitrary shapes and \\ unequal sizes. \\ - Does not require a priori \\ specification of the \\ number of clusters.
}

converges to a final, stable assignment. We ran several iterations of the k-medoids algorithm using Euclidean distance and varying $k$ between 5 and 10 . We calculated the average silhouette width for each solution and selected the $k$ that yielded the solution with the highest average silhouette width.

\section{Density-Based Clustering}

For density-based clustering, we used the OPTICS algorithm ${ }^{27}$, ${ }^{28}$. OPTICS requires a specified minimum number of nearest neighbors (MinPts) within a specified radius around the observation $(\varepsilon)$. Points located in relatively low-density regions have few "neighbors" and may be classified as outliers. We set MinPts at $1 \%$ of the study population, or 62 patients (observations), to help ensure that clusters would not be so small as to be operationally insignificant. Given a specified $\varepsilon$, OPTICS returns any cluster solution corresponding to a radius less than $\varepsilon$. We varied $\varepsilon$ such that we extracted all of the clustering solutions that produced between five and ten clusters. Then, we then calculated the average silhouette width for each solution and selected the solution with the highest average silhouette width.

\section{Evaluating Algorithm Performance}

First, we performed a visual examination of the cluster assignments using the two-dimensional representation of the data set generated by the t-SNE algorithm. We created colored hull plots to visualize the clusters generated by each algorithm.
Second, we employed a set of ridge regression ${ }^{29}$ models to better understand the relationship between cluster assignment and clinical variables. A separate set of models was implemented for each cluster. The dependent variable in each model was a dichotomous indicator of assignment to a given cluster, and the original 161 variables were independent variables. The magnitudes of the resulting model coefficient estimates represent the relative contribution of each independent variable to discriminating patients in one cluster from other high-cost patients. Details of model fitting are provided in Appendix 6, including technical details about how we averaged across models and how we chose penalization terms to ensure comparability across clusters and methods. Following model fitting, we computed the range of the estimated coefficients (maximum value minus minimum value) across clusters for each independent variable. This metric is a measure of variable importance, where a large value suggests that the variable is useful for distinguishing observations in one cluster from the rest. In order to visualize these results, we plotted the values of the variables with the largest 20 ranges for each of the clustering methods.

Finally, we examined the extent to which cluster assignments were associated with differences in utilization and spending. As noted above, these variables were not used for clustering. First, we calculated intra-cluster averages for the following utilization and spending variables: inpatient hospital (IP) admissions; total number of IP days; emergency department (ED) visits; total spending; the percentage of spending 
attributable to inpatient care, medications, and other sources; and the percentage of persistently high-cost patients (those who remained in the top decile of spending the subsequent year). Next, we calculated inter-cluster variance in these utilization and spending averages for each clustering method.

\section{Implementation Details}

Data preparation was done in SAS version 9.4 (SAS Institute, Cary, NC). R version 3.2.5 was used for all other analyses. The Rtsne package version 0.13 was used for dimension reduction and the dbscan package version 1.1-1 was used for OPTICS density-based clustering analysis. The cluster package version 2.0.7-1 was used for agglomerative hierarchical clustering with Ward's criterion and k-medoids clustering.

\section{RESULTS}

Figure 1 summarizes the results of each clustering algorithm. Connectivity-based clustering identified eight subgroups of high-cost patients, ranging in size from 458 to 1170 patients. Centroid-based clustering identified five subgroups, ranging in size from 1003 to 1427 patients. Density-based clustering identified ten subgroups, ranging in size from 56 to 3686 patients, with 382 not assigned to any subgroup.

Figure 1 also graphically depicts the clustering results in the two-dimensional projection of our dataset generated by the tSNE dimension reduction algorithm. Each point is a patient in the study population, and the distance between two points approximates their similarity in high-dimensional space. Colored hull plots are used to depict the subgroups of patients identified by each clustering method. Connectivity- and centroid-based clustering identified subgroups of roughly similar sizes and shapes. Density-based clustering, on the other hand, yielded subgroups of markedly varied sizes and shapes. Figure 1 also highlights the difference between the three clustering methods with respect to the treatment of outliers. The density-based clustering algorithm permitted outliers, whereas the connectivity- and centroid-based algorithms did not. By forcing all points into clusters, most of the subgroups identified by the connectivity- and centroid-based algorithms appear more heterogeneous.

Results from the ridge regression analysis are summarized in Figure 2, which depicts the estimated coefficient ranges for the 20 variables with the ranges among each clustering method. This range roughly corresponds to the extent to which the variable contributes to differentiation among subgroups. Centroid-based clustering had the largest range for the top ranked variable. Density-based clustering resulted in a larger range for the next 11 highest ranked variables, after which the three methods converged. The estimated coefficient ranges for each variable, as well as a list of the variables depicted in Figure 2, are provided in Appendix 8.
Variance of utilization and spending measures across subgroups is summarized in Table 2. Higher variance corresponds to more differentiation across subgroups. Density-based clustering resulted in subgroups with the greatest variance across all utilization and spending variables, with the exception of ED visits. The difference was most pronounced among IP admissions, IP days, total spending, and the percent of spending attributable to medications. The mean values from which variances were calculated are provided in Appendix 9.

\section{DISCUSSION}

We evaluated the performance of three different clustering methods for identifying subgroups of high-cost patients using a multi-dimensional clinical and administrative data set. Specifically, we compared connectivity-based clustering using agglomerative hierarchical clustering, centroid-based clustering with the k-medoids algorithm, and density-based clustering with the OPTICS algorithm.

We performed several analyses to assess the performance of the three clustering algorithms. Our intention was to evaluate the capability of each algorithm to identify subgroups of patients that were (1) clinically distinct and (2) associated with meaningful differences in relevant utilization metrics. To answer the first question, we fit a set of post hoc ridge regression models to the subgroups in order to learn about how patients in one subgroup were different from the rest of the population. We then compared the estimated model coefficients for each variable across subgroups. This allowed us to determine the extent to which specific variables (i.e., clinical characteristics) created differentiation across subgroups. In general, we found that the estimated coefficient ranges for the density-based algorithm were greater than those for the connectivity- and centroidbased algorithms, suggesting that the subgroups identified by the density-based algorithm were the most clinically distinct.

To answer the second question, we examined the variance of selected utilization and spending measures across subgroups. The level of variance across subgroups identified by the density-based clustering algorithm exceeded that of the connectivity- and centroid-based algorithms for nearly all measures. High variance across utilization and spending measures has important operational implications. For example, a subgroup with high rates of inpatient admissions will benefit from different care management interventions than those necessary for a subgroup with high rates of spending attributable to medications.

Since utilization and spending variables were not used for clustering, this analysis also functioned as a test of external validity. For all three clustering methods, we found significant variance in utilization and spending variables across subgroups. This suggests that clustering patients on clinical variables can effectively identify unique subgroups of patients with distinct patterns of utilization and spending. 


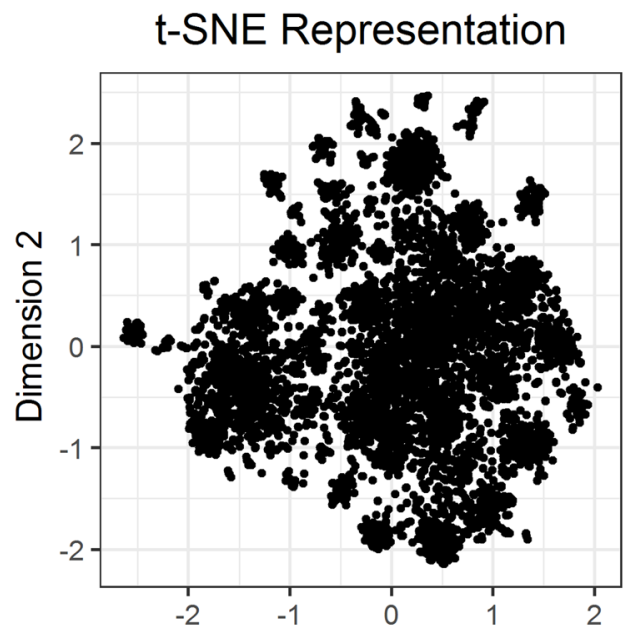

Centroid-Based Clustering

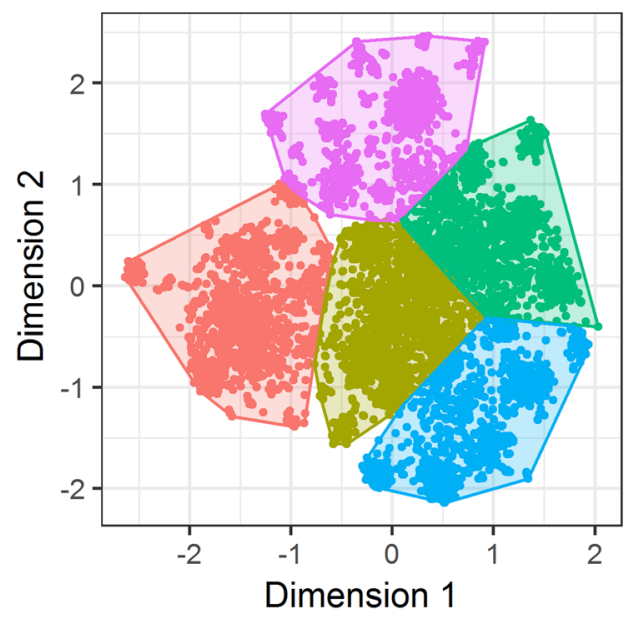

Connectivity-Based Clustering

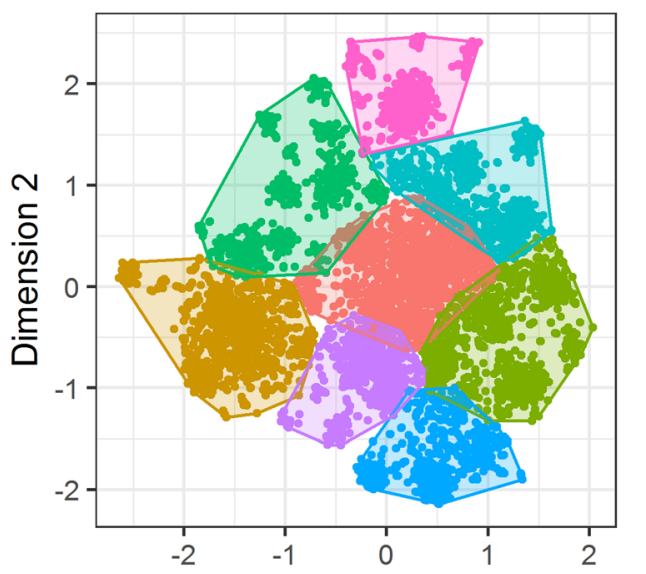

- $\mathrm{n}=1170$

- $\mathrm{n}=1018$

- $n=954$

- $n=723$

- $n=701$

- $n=578$

- $n=552$

- $n=458$
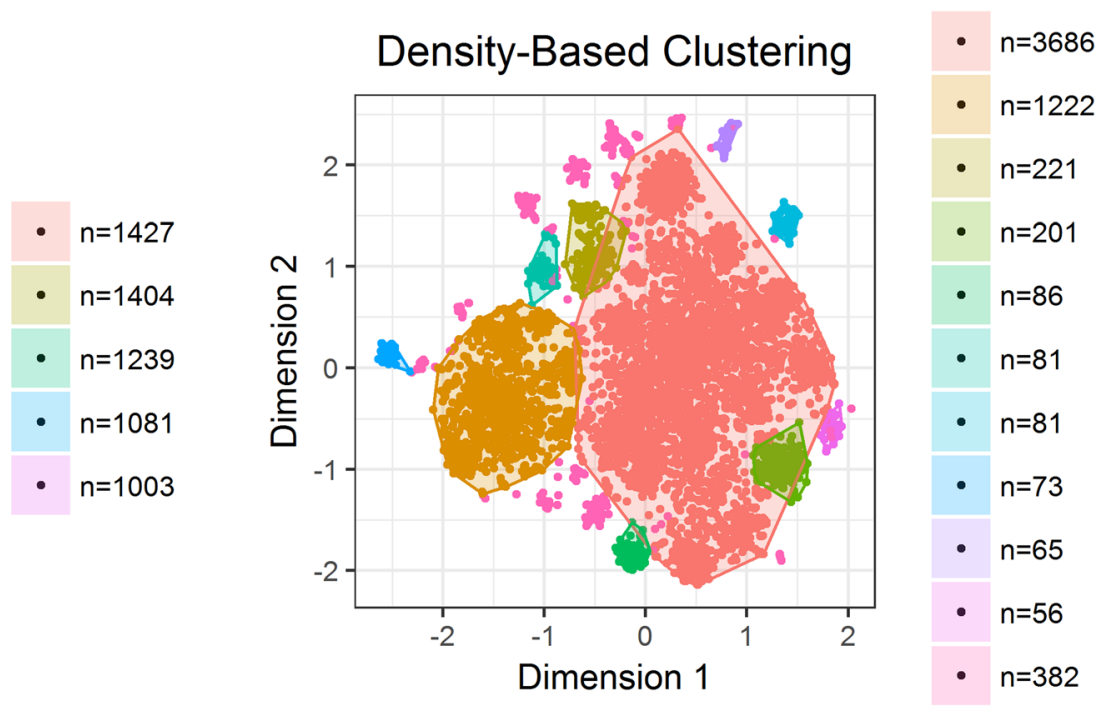

Figure 1 Visual representation of patient subgroups, by clustering method. The figures represent two-dimensional projection of our dataset generated by the t-SNE dimension reduction algorithm (t-SNE projection). Each point is a patient in the study population, and the distance between two points approximates their similarity in high-dimensional space. The top left plot represents the t-SNE projection in insolation. The remaining plots overlay colored convex cluster outlines to the t-SNE projection for each of the three clustering methods. The number of patients in each cluster is provided next to the plots.

Taken together, our results suggest that density-based clustering with the OPTICS algorithm performs best for identifying clinically distinct and operationally significant subgroups of high-cost patients in our data, and may be a promising and feasible approach for subgroup analysis in health plan or health system data. An accompanying article provides an indepth description of the clinical composition, utilization patterns, and spending trajectories of the subgroups identified by the OPTICS algorithm ${ }^{19}$.

Differences in the algorithmic approach of density-based clustering with OPTICS may explain why it outperformed connectivity- and centroid-based algorithms in this study. First, OPTICS allows for "noise" points (i.e., observations not assigned to any cluster), whereas connectivity- and centroid-based clustering algorithms force all observations into clusters. By forcing outlier observations into subgroups, the resultant subgroups become more heterogeneous. Since outliers are frequent in medical data, this may limit clinical utility. We found that including outliers (as defined by OPTICS) led to subgroups that were less clinically distinct and, therefore, less operationally significant. Second, the OPTICS algorithm is able to identify subgroups of uneven sizes, as demonstrated in Figure 1. In care management settings, programs are often designed for various patient groups, and the size of sub-populations to which the programs apply may vary drastically. Lastly, OPTICS does not require one to specify the number of clusters a priori, an attractive property in clinical settings where the underlying data structure may not be well understood. However, the process of tuning models to maximize discrimination and select the optimal number of clusters may mitigate this advantage.

Using unsupervised machine learning algorithms to identify subgroups of high-cost patients is a departure from existing efforts to segment high-cost populations that rely predominately on expert-opinion derived taxonomies ${ }^{1,3,5,6,30}$. We do not expect, nor advocate, that clustering and other machine 


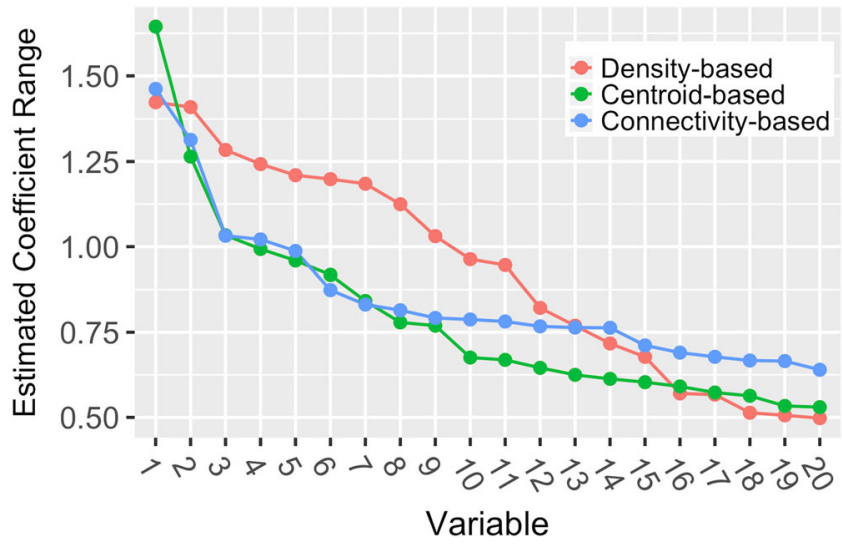

Figure 2 Summary of the results of the ridge regression analysis. The ridge regression estimated coefficient range represents the range of estimated coefficients (maximum value minus minimum value) for a given variable, across all subgroups. These ranges correspond to the extent to which a variable drives differentiation among the subgroups of patients identified by that method-a large value suggests that the variable is useful for distinguishing observations in one subgroup from the rest. For each clustering method, the 20 variables with the largest coefficient ranges are included. A list of the variables depicted along the $x$-axis for each method is provided in Appendix 8.

learning methods replace existing, expert-opinion derived taxonomies. Rather, these methods could serve as an important complement through two major channels. First, for payers and large delivery organizations, there likely exists sufficient inhouse technical expertise to use clustering to derive high-cost patient subgroups directly from internal data sets. This approach, which utilizes free, publicly available algorithms and software packages, contrasts with the recent proliferation of proprietary, "black box" commercial risk-prediction and population segmentation algorithms. Second, researchers and policy analysts may be able to use clustering to identify common subgroups across populations that could be added to existing taxonomies.

This study has several limitations. First, by pre-specifying a small number of subgroups (five to ten), we may have failed to identify a more quantitatively optimal segmentation of the data

Table 2 Variance of Utilization and Spending Variables Across Subgroups, by Clustering Method

\begin{tabular}{llll}
\hline \hline & $\begin{array}{l}\text { Connectivity- } \\
\text { based clustering }\end{array}$ & $\begin{array}{l}\text { Centroid- } \\
\text { based } \\
\text { clustering }\end{array}$ & $\begin{array}{l}\text { Density- } \\
\text { based } \\
\text { clustering }\end{array}$ \\
\hline $\begin{array}{l}\text { Inpatient } \\
\text { admissions }\end{array}$ & 0.55 & 0.51 & 0.77 \\
$\begin{array}{l}\text { Inpatient days } \\
\text { ED visits }\end{array}$ & 5.23 & 5.17 & 7.17 \\
Total spending, & 0.86 & 0.88 & 0.78 \\
$\begin{array}{l}\text { Inpatient, \% } \\
\text { Medication, \% }\end{array}$ & 11,887 & 12,441 & 14,621 \\
$\begin{array}{l}\text { Other, \% } \\
\text { Persistently }\end{array}$ & $14 \%$ & $12 \%$ & $18 \%$ \\
high-cost, \% & $20 \%$ & $5 \%$ & $22 \%$ \\
\hline
\end{tabular}

Persistently high-cost was defined as remaining in the highest decile of spending in the subsequent year. The mean values from which variances were calculated are provided in Appendix 9

ED emergency department set that included several small, homogeneous subgroups. However, we selected this range based on prior literature ${ }^{1,3,5}$ and to avoid identifying subgroups so small that they would be operationally insignificant. Second, our comparison of different clustering methods used illustrative algorithms from each class of methods and was limited to three classes of methods. This should not be construed to be a comprehensive, or definitive, assessment of clustering approaches. We chose to focus on three of the most common clustering methods that can be implemented with standard packages in open source software. Finally, we limited our study to a single approach to dimensional reduction, t-SNE. Although t-SNE has been shown to produce well-separated clusters in a variety of biomedical settings ${ }^{31-34}$, it is a stochastic method that relies on a number of user-specified inputs. Future work should include comparisons of t-SNE to other projection techniques, such as principal component analysis (PCA).

Corresponding Author: Amol S. Navathe, $M D, P h D$; Department of Medical Ethics and Health PolicyUniversity of Pennsylvania Perelman School of Medicine, 1108 Blockley Hall, Philadelphia, PA 19104, USA (e-mail:amol@wharton.upenn.edu).

Funding Information This study was supported by a grant from the Anthem Public Policy Institute and, in part, under a grant with the Pennsylvania Department of Health.

\section{Compliance with Ethical Standards:}

This study was approved by the Institutional Review Board of the University of Pennsylvania.

Conflict of Interest: Dr. Navathe reports that he has received grant support from Hawaii Medical Service Association, Anthem Public Policy Institute, and Oscar Health; personal fees from Navvis and Co, Navigant Inc., Lynx Medical, Indegene Inc., Agathos, Inc, and Sutherland Global Services; personal fees and equity from NavaHealth; serves on the board without compensation for Integrated Services, Inc., speaking fees from the Cleveland Clinic, and honoraria from Elsevier Press. Dr. Linn reports that she has received grant support from Hawaii Medical Service Association. Dr. Jain reports employment by Anthem, Inc.; stock ownership in Anthem, Inc., and honoraria from Elsevier Press. Ms. Kowalski reports employment by Anthem, Inc. and stock ownership in Anthem, Inc. and Amazon. Dr. Powers reports employment by Anthem, Inc. All other authors declare no conflicts of interest.

Disclaimer: The Pennsylvania Department of Health specifically disclaims responsibility for any analyses, interpretations, or conclusions.

Publisher's Note Springer Nature remains neutral with regard to jurisdictional claims in published maps and institutional affiliations.

\section{REFERENCES}

1. National Academy of Medicine. Effective Care for High-Need Patients. Washington, DC: National Academy of Medicine; 2017.

2. Hong CS, Siegel AL, Ferris TG. Caring for High-Need, High-Cost Patients: What Makes for a Successful Care Management Program? 2014; https://www.commonwealthfund.org/sites/default/files/documents/_media_files_publications_issue_brief_2014_aug_1764_hong_ caring_for_high_need_high_cost_patients_ccm_ib.pdf. Accessed October 19, 2018. 
3. Joynt KE, Figueroa JF, Beaulieu N, Wild RC, Orav EJ, Jha AK Segmenting high-cost Medicare patients into potentially actionable cohorts. Healthc (Amst). 2017;5(1-2):62-67.

4. Blumenthal D, Abrams MK. Tailoring Complex Care Management for High-Need, High-Cost Patients. JAMA 2016;316(16):1657-1658.

5. Clough JD, Riley GF, Cohen M, et al. Patterns of care for clinically distinct segments of high cost Medicare beneficiaries. Healthc (Amst). 2016;4(3):160-165.

6. Lynn J, Straube BM, Bell KM, Jencks SF, Kambic RT. Using population segmentation to provide better health care for all: the "Bridges to Health" model. Milbank Q. 2007;85(2):185-208; discussion 209-112.

7. Berkhin P. A Survey of Clustering Data Mining Techniques. In: Kogan J, Nicholas C, Teboulle M, eds. Grouping Multidimensional Data: Recent Advances in Clustering. Berlin, Heidelberg: Springer Berlin Heidelberg; 2006:25-71.

8. Gan G, Ma C, Wu J. Data Clustering: Theory, Algorithms, and Applica tions. Society for Industrial and Applied Mathematics; 2007.

9. Moore WC, Meyers DA, Wenzel SE, et al. Identification of asthma phenotypes using cluster analysis in the Severe Asthma Research Program. Am J Respir Crit Care Med. 2010;181(4):315-323.

10. Haldar P, Pavord ID, Shaw DE, et al. Cluster analysis and clinical asthma phenotypes. Am J Respir Crit Care Med. 2008;178(3):218-224.

11. Weatherall M, Shirtcliffe P, Travers J, Beasley R. Use of cluster analysis to define COPD phenotypes. Eur Respir J. 2010;36(3):472-474.

12. Chen CZ, Wang LY, Ou CY, Lee CH, Lin CC, Hsiue TR. Using cluster analysis to identify phenotypes and validation of mortality in men with COPD. Lung. 2014;192(6):889-896.

13. Ahmad T, Pencina MJ, Schulte PJ, et al. Clinical implications of chronic heart failure phenotypes defined by cluster analysis. J Am Coll Cardiol 2014;64(17):1765-1774.

14. Ahmad T, Desai N, Wilson F, et al. Clinical Implications of Cluster Analysis-Based Classification of Acute Decompensated Heart Failure and Correlation with Bedside Hemodynamic Profiles. PloS one. 2016;11(2):e0145881.

15. Erro R, Vitale C, Amboni M, et al. The heterogeneity of early Parkinson's disease: a cluster analysis on newly diagnosed untreated patients. PloS one. 2013;8(8):e70244.

16. Hamid JS, Meaney C, Crowcroft NS, Granerod J, Beyene J, Group UKEoES. Cluster analysis for identifying sub-groups and selecting potential discriminatory variables in human encephalitis. BMC Infect Dis. 2010;10:364
17. Newcomer SR, Steiner JF, Bayliss EA. Identifying subgroups of complex patients with cluster analysis. Am J Manag Care. 2011;17(8):e324-332.

18. Lee NS, Whitman N, Vakharia N, Ph DG, Rothberg MB. High-Cost Patients: Hot-Spotters Don't Explain the Half of It. $J$ Gen Intern Med 2017;32(1):28-34

19. Powers BW, Yan J, Zhu J, et al. Subgroups of High-Cost Medicare Advantage Patients: An Observational Study. J Gen Intern Med 2018.

20. Bellman R. Adaptive control processes: a guided tour. Princeton, N.J., Princeton University Press; 1961.

21. Donoho DL. High-dimensional data analysis: The curses and blessings of dimensionality. AMS Math Challenges Lecture. 2000:1-32.

22. Van Der Maaten L, Hinton G. Visualizing data using t-SNE. J Mach Learn Res 2008;9(Nov):2579-2605.

23. Van Der Maaten L. Accelerating t-SNE using tree-based algorithms. $J$ Mach Learn Res 2014;15(1):3221-3245.

24. Rousseeuw PJ. Silhouettes: A graphical aid to the interpretation and validation of cluster analysis. J Comput Appl Math 1987;20:53-65.

25. Ward JH. Hierarchical Grouping to Optimize an Objective Function. J Am Stat Assoc 1963;58(301):236-244.

26. Kaufman L, Rousseeuw PJ. Clustering by means of medoids. Amsterdam: North-Holland/Elsevier; 1987.

27. Ester M, Kriegel H-P, Sander J, Xu X. A density-based algorithm for discovering clusters a density-based algorithm for discovering clusters in large spatial databases with noise. Proceedings of the Second International Conference on Knowledge Discovery and Data Mining; 1996; Portland, Oregon.

28. Ankerst M, Breunig MM, Kriegel H-P, Sander J. OPTICS: ordering points to identify the clustering structure. SIGMOD Rec. 1999;28(2):49-60.

29. Hoerl AE, Kennard RW. Ridge Regression: Biased Estimation for Nonorthogonal Problems. Technometrics. 1970;12(1):55-67.

30. Figueroa JF, Jha AK. Approach for Achieving Effective Care for High-Need Patients. JAMA Intern Med. 2018;178(6):845-846.

31. Grun D, Lyubimova A, Kester L, et al. Single-cell messenger RNA sequencing reveals rare intestinal cell types. Nature. 2015;525(7568):251-255.

32. Keren-Shaul H, Spinrad A, Weiner A, et al. A Unique Microglia Type Associated with Restricting Development of Alzheimer's Disease. Cell. 2017;169(7):1276-1290 e1217.

33. Becher B, Schlitzer A, Chen J, et al. High-dimensional analysis of the murine myeloid cell system. Nat Immunol. 2014;15(12):1181-1189.

34. Abdelmoula WM, Balluff B, Englert S, et al. Data-driven identification of prognostic tumor subpopulations using spatially mapped t-SNE of mass spectrometry imaging data. Proc Natl Acad Sci $U$ S A. 2016;113(43): 12244-12249. 